\title{
COVID-19 Vaccine-Induced Thrombosis and Thrombocytopenia: First Confirmed Case from India
}

\author{
Kundan Mishra $^{1} \cdot$ Satish Barki $^{2} \cdot$ Somali Pattanayak $^{3} \cdot$ Madabhushi Shyam $^{4} \cdot$ \\ Amit Sreen $^{2} \cdot$ Suman Kumar $^{1} \cdot$ Jyoti Kotwal $^{5}$
}

Received: 11 August 2021/Accepted: 18 September 2021/Published online: 29 September 2021

(C) Indian Society of Hematology and Blood Transfusion 2021

Keywords COVID-19 · Covishield · Thrombosis · VITT · Anti-PF4 Ab

\section{Dear Editor,}

An 18-year-old boy was brought to the emergency with complaints of headache and recurrent vomiting for five days. His attendants also gave a history of excessive drowsiness for the last two days. He had no significant past medical or surgical history. There was a history of vaccination (Covishield, 1st dose) 17 days before the presentation. Clinically, he was afebrile, had a pulse rate of $80 \mathrm{bpm}$, and blood pressure 120/76 mm of $\mathrm{Hg}$. He was drowsy but arousable. He was producing incomprehensible sounds and was able to localize the pain. He had gaze preference to the left side. Extensor plantar response was noted on the right side. An

Suman Kumar

hematologyhod@gmail.com

1 Department of Clinical Hematology and Stem Cell Transplant, Medical Division Army Hospital (Research \& Referral), Delhi, India 110010

2 Department of Neurosciences, Army Hospital (Research \& Referral, Delhi, India

3 Department of Radio-Diagnosis, Army Hospital (Research \& Referral, Delhi, India

4 Department of Anesthesiology and Critical Care, Army Hospital (Research \& Referral, Delhi, India

5 Department of Hematology, Sir Ganga Ram Hospital, Delhi, India urgent computed tomography (CT) imaging of the head showed hyperdense lesions involving the left parieto-temporal region suggestive of intracerebral hemorrhage (Fig. 1a). The patient was treated with mannitol, dexamethasone, phenytoin, and supportive care.

His routine evaluation showed, hemoglobin $13.1 \mathrm{~g} / \mathrm{dL}$, WBC $17,250 / \mathrm{cmm}$ and platelet $85,000 / \mathrm{cmm}$. His creatinine was $0.6 \mathrm{mg} / \mathrm{dL}$ and AST and ALT were 107 and 166U/L respectively. Coagulation parameters (PT, aPTT and fibrinogen) were normal with elevated D-dimer $(8334.8 \mathrm{ng} /$ $\mathrm{mL}$ ). During the next $12-\mathrm{h}$, his neurological status continued to deteriorate and for airway protection, he was electively intubated. Because of progression on anti-edema measures and history of vaccination, and thrombocytopenia, clinical diagnosis of vaccine-induced thrombosis and thrombocytopenia (VITT) was considered. The patient was taken out for a computed tomography (CT) venography head and contrast enhanced CT (CECT) chest and abdomen. The venography images showed a filling defect in the superior sagittal sinus, and bilateral sigmoid sinuses (right $>$ left) (Fig. 1b). The CECT abdomen images showed a filling defect in the portal vein (Fig. 1c). The Gel card test for Anti-PF4/heparin complex antibody was positive and the heparin-induced platelet aggregationbased functional assay for HIT antibodies was confirmative of the presence of heparin dependent platelet aggregation (Figure-1d-e). Following these results, the diagnosis was confirmed as VITT, and fondaparinux was started. However, the patient's general condition continued to deteriorate, and he succumbed to his illness on day seven of hospitalization.

Coronavirus disease 2019 (COVID-19) pandemic begun on 27th January 2020 in India. Since then more than four lacs people have lost their life [1]. Vaccination against the coronavirus is the most effective tool to control the 


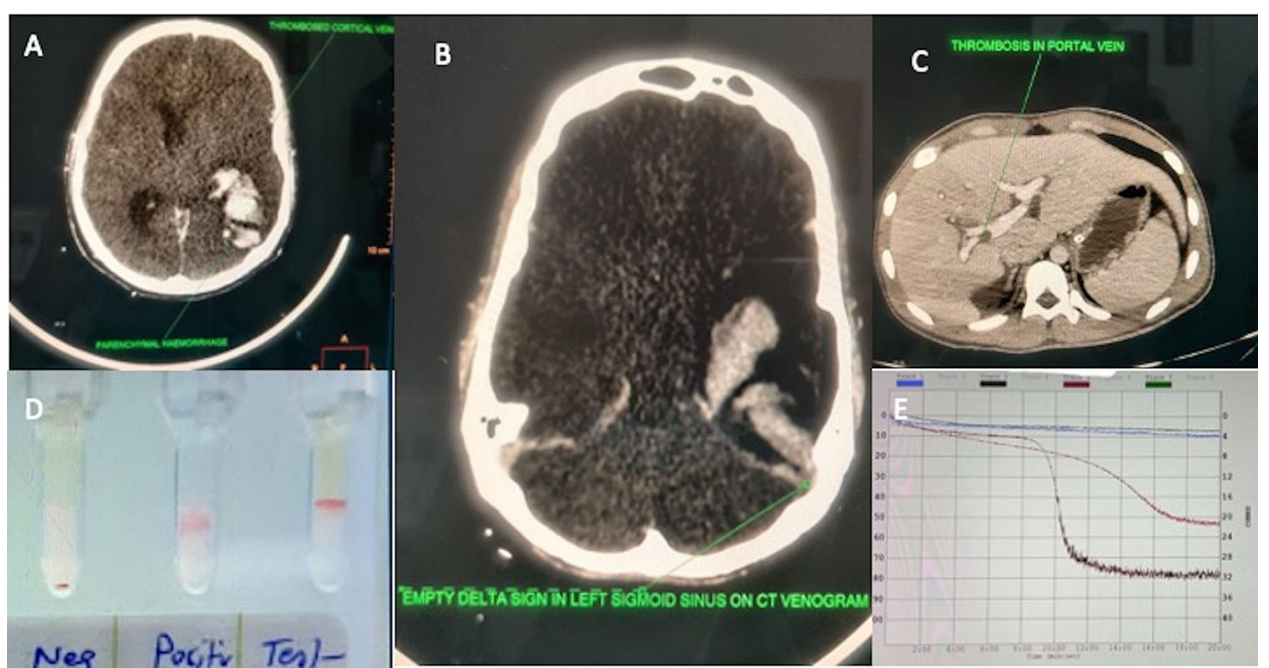

Fig. 1 a Computed tomography (CT) images of the head showing hyperdense lesions involving the left parieto-temporal region suggestive of intracerebral hemorrhage, b CT venography images showed a filling defect in the superior sagittal sinus, and bilateral sigmoid sinus (right $>$ left), $\mathbf{c}$ the CECT abdomen images also showed a filling defect in the portal vein. d An Image of the Gel card test showing positive test (patient) for Anti-PF4/heparin complex antibody. e An image showing the graph of platelet aggregation based functional assay for HIT antibodies (Patients serum does show

COVID-19 pandemic. Increase vaccination coverage in the community protects even the unvaccinated individuals through herd immunity. In India, Covishield (ChAdOx1 nCoV-19) has been the most widely used vaccine since it was given emergency approval by the government of India on 03 January 2021 [2]. Covishield is an adenovirus-based, non-replicating vaccine and has shown good efficacy and safety profile in clinical trials. Since, vaccination with, Covishield (ChAdOx1 nCoV-19) started, there have been reports of thrombocytopenia and thrombosis (VITT) in recipients [3]. In a patient who has a history of vaccination, VITT is suspected based on new-onset thrombocytopenia, and thrombosis developing within 5-20 days of vaccination and further confirmed by the presence of anti-PF4 antibodies. The proposed mechanism is heparin-independent anti-PF4 autoantibody-mediated activation of the coagulation pathway [4]. A high degree of suspicion is required to diagnose this condition. Treatment is recommended with Xa inhibitors (rivaroxaban, apixaban) or fondaparinux along with intravenous immunoglobulin (IVIG). Our case emphasizes the need for more awareness regarding this rare entity following vaccination. Moreover, in developing countries like India, where high-quality laboratory and radiological facilities are not readily available, awareness is the only tool that will expedite the diagnosis and save the patients. There has not been any increased aggregation of the donor (control platelet) with 0.5 and $1.0 \mathrm{U} / \mathrm{ml}$ heparin dosage (low dose heparin). There is no aggregation with control platelets with the patient's serum in the absence of heparin as well as with high dose heparin. Aggregation of more than $20 \%$ is taken as positive for heparin-induced platelet activation. In this case, the platelet aggregation is $82 \%$ confirming the presence of heparin-dependent platelet aggregation in the case of VITT following Adenovector COVID-19 vaccination)

official case of VITT in India [5]. Therefore, the index case may be the first case of VITT from India.

Funding This research received no specific grant from any funding agency, commercial or not-for-profit sectors.

\section{Declarations}

Conflict of interest The author declares that they have no conflict on interest.

Informed Consent Informed signed written consent was taken from the patient involved.

Ethical Approval All procedures performed in studies involving human participants were in accordance with the ethical standards of the institutional and/or national research committee and with the 1964 Helsinki declaration and its later amendments or comparable ethical standards.

Human and Animals Rights No animals were involved in the study.

\section{References}

1. MOHFW COVID-19 Report India. 2021. Available from: https:// main.mohfw.gov.in. Accessed July 07, 2021

2. HFW_DCGI_energency_use_authorisation_03012021_2.pdf. 2021. Available from: https://www.icmr.gov.in/pdf/press_rea lease_files/HFW_DCGI_energency_ 03012021_2.pdf. Accessed July 07, 2021 use_authorisation_ 
3. Greinacher A, Thiele T, Warkentin TE, Weisser K, Kyrle PA, Eichinger S (2021) Thrombotic Thrombocytopenia after ChAdOx1 nCov-19 vaccination. N Engl J Med 384(22):2092-2101

4. Franchini M, Liumbruno GM, Pezzo M (2021) COVID-19 vaccine-associated immune thrombosis and thrombocytopenia (VITT): diagnostic and therapeutic recommendations for a new syndrome. Eur J Haematol 107(2):173-180

5. MOHFW adverse event following immunization report India Covishield vaccine. 2021. Available from: https://main.mohfw.
gov.in/Organisation/Departments-of-Health-and-Family-Welfare/ immunization/aefi-reports. Accessed July 07, 2021

Publisher's Note Springer Nature remains neutral with regard to jurisdictional claims in published maps and institutional affiliations. 